\title{
Erratum
}

\section{Geometric finiteness theorems via controlled topology}

\author{
Karsten Grove ${ }^{1}$, Peter Petersen $V^{2}$, and Jyh-Yang Wu ${ }^{1}$ \\ ${ }^{1}$ University of Maryland, Department of Mathematics, College Park, Maryland 20742, USA \\ 2 UCLA, Department of Mathematics, Los Angeles, California 90024, USA
}

Oblatum 15-VI-1990

There is a mistake in the proof of the main theorem, which is carried over from [PV]. We shall reformulate the main theorem here, so as to make it correct and also show that the geometric theorems A, B, and C hold as stated.

Notation and references are as in the paper. The error occurs in Theorem 1.2 where it is stated that the class of compact LGC $(\rho)$ metric spaces of dimension $\leqq n<\infty$ is closed in the Gromov-Hausdorff topology. It was kindly pointed out to us by S. Ferry and T. Engel, that in fact there is a sequence $\left\{M_{i}\right\}$ of compact $\operatorname{LGC}(\rho)$ metric $n$-manifolds, such that $\lim M_{i}$ exists and is infinite dimensional $[\mathrm{E}]$. We would also like to mention that although our proof of Theorem 1.4 is based in part on 1.2, it remains correct as stated (cf. [F]).

In order to restate the theorems we need an additional definition. A compact metric space $X$ is said to have $N:(0, a] \rightarrow \mathbb{R}_{+}$as a covering-function, provided $X$ can be covered by $\leqq N(\varepsilon) \varepsilon$-balls for every $\varepsilon \in(0, a]$. The crucial observations are then:

Proposition 1 (cf. [G1] and 1.5) Given a function $N:(0, a] \rightarrow \mathbb{R}_{+}$. The space $\mathscr{M}$ of compact metric spaces $X$ having $N$ as covering function is compact in the Gromov-Hausdorff topology.

Proposition 2 (cf. [PS]) Suppose $N:(0, a] \rightarrow \mathbb{R}_{+}$satisfies $\lim \sup \varepsilon^{-\alpha} N(\varepsilon)<\infty$ for some $\alpha>0$. Then any compact metric space $X$ which has $N$ as a covering function must have Hausdorff dimension $\leqq \alpha$. In particular $\operatorname{dim} X \leqq[\alpha]$.

We now fix a concave function $\rho:[0, R] \rightarrow[0, \infty)$ with $\rho(0)=0, \rho(\varepsilon) \geqq \varepsilon$ for all $\varepsilon \in[0, R]$, and a function $N:(0, a] \rightarrow \mathbb{R}_{+}$with $\lim \sup \varepsilon^{-n} N(\varepsilon)<\infty$. With this terminology we replace Theorem 1.2 by: $\varepsilon \rightarrow 0$

Theorem 1.2 The class of compact metric spaces which are LGC $(\rho)$ and have $N$ as a covering function is compact in the Gromov-Hausdorff topology. Moreover, any space $X$ in this class has $\operatorname{dim} X \leqq n$.

Proof. Let $\left\{X_{i}\right\}$ be a sequence of compact $\operatorname{LGC}(\rho)$ metric spaces having $N$ as a covering function. From Proposition $1\left\{X_{i}\right\}$ has an accumulation point 
$X$, which also has $N$ as a covering function. Then $\operatorname{dim} X \leqq n$ according to Proposition 2. We can now use either [Bo] or [PV] to conclude that also $X$ is $\operatorname{LGC}(\rho)$. q.e.d.

The corresponding change in the main theorem is reflected in:

Main Theorem The class of closed metric n-manifolds, $n \neq 3$, which are LGC $(\rho)$ and have $N$ as a covering function contains at most finitely many homeomorphism types.

Proof. Let $\left\{M_{i}\right\}$ be a sequence of closed LGC $(\rho)$ metric $n$-manifolds having $N$ as a covering function. By 1.2 we can assume that $\left\{M_{i}\right\}$ converges to a compact metric space $X$ which is also LGC $(\rho)$ and has $\operatorname{dim} X \leqq n$. It then follows from 1.3 that in fact $\operatorname{dim} X=n$. The remaining part of the proof is unchanged (cf. the proofs of 2.1, 2.2 and 3.1 of the paper). q.e.d.

Corollary 3.3 holds with the obvious modification that $\mathscr{M}\left(n, C_{R}\right)$ be a class of closed riemannian $n$-manifolds with criticallity radius $\geqq R$ and $N(\varepsilon)=C \varepsilon^{-n}$ as a covering function.

It remains to be seen that the classes of manifolds in Theorems $\mathrm{A}$ and $\mathrm{C}$ satisfy the hypotheses of the main theorem above. In fact, the only thing missing is to explain why these classes have covering functions. This, however, is contained in the following two lemmas which are basically well known.

Lemma 1 Fix an integer $n \geqq 2, a$ real $k$ and $D>0$. There exists a constant $C$ $=C(n, k, D)$ so that any closed riemannian $n$-manifold $M$ with ric $M \geqq(n-1) k$ and diam $M \leqq D$ has $N(\varepsilon)=C \cdot \varepsilon^{-n}:(0, D] \rightarrow \mathbb{R}_{+}$as a covering function.

Proof. By standard use of relative volume comparison (as in [GP 1]) we know that

$$
N(\varepsilon)=\int_{0}^{D} \sinh ^{n-1}(\sqrt{|k|} t) d t / \int_{0}^{e / 2} \sinh ^{n-1}(\sqrt{|k|} t) d t
$$

is a covering function. But $\sinh (\sqrt{|k|} t) \geqq \sqrt{|k|} t$ and therefore

$$
N(\varepsilon) \leqq n \cdot 2^{n} \cdot|k|^{(1-n) / 2}\left(\int_{0}^{D} \sinh ^{n-1}(\sqrt{|k|} t) d t\right) \cdot \varepsilon^{-n} \text {. q.e.d. }
$$

Lemma 2 Given an integer $n \geqq 2$ and positive numbers $i$ and $V$. There is a constant $C^{\prime}=C^{\prime}(n, i, V)$ so that any closed riemannian $n$-manifold $M$ with inj $M \geqq i$ and vol $M \leqq V$ has $N(\varepsilon)=C^{\prime} \cdot \varepsilon^{-n}:(0, i / 2] \rightarrow \mathbb{R}_{+}$as a covering function.

Proof. When $\varepsilon \leqq i / 2$ it follows from [Cr] that vol $B(p, \varepsilon) \geqq c(n) \varepsilon^{n}$ for some universal constant $c(n)$ independent of $p \in M$. Thus if $B\left(p_{1}, \varepsilon / 2\right), \ldots, B\left(p_{N}, \varepsilon / 2\right)$ is a maximal collection of disjoint $\varepsilon / 2$-balls, we get

$$
N \leqq \operatorname{vol} M / c(n)(\varepsilon / 2)^{n} \leqq c(n)^{-1} \cdot 2^{n} \cdot V \cdot \varepsilon^{-n} .
$$

But $B\left(p_{1}, \varepsilon\right), \ldots, B\left(p_{N}, \varepsilon\right)$ obviously covers $M$ and so

$$
N(\varepsilon)=c(n)^{-1} \cdot 2^{n} \cdot V \cdot \varepsilon^{-n}:(0, i / 2] \rightarrow \mathbb{R}_{+}
$$

is a covering function. q.e.d.

\section{Additional References}

[E] Engel, T.: Deformation and rigidity along paths of manifolds Thesis. Binghamton: Suny 1990

[F] Ferry, S.: An extension of simple-homotopy theory. Abstr. Pap. Am. Math. Soc. vol. 11, No. 3, 857-55-44, 254 (1990)

[PS] Pontrjagin, L., Schnirelmann, L.: Sur une propriété métrique de la dimension. Ann. Math., II. Ser. 33, 152-162 (1932) 